\title{
A Comparison between MLP and SVR Models in Prediction of Thermal Properties of Nano Fluids
}

\author{
R. Kavitha ${ }^{1 \dagger}$ and P. C. Mukesh Kumar ${ }^{2}$ \\ ${ }^{1}$ Parisutham Institute of Technology and Science, Thanjavur-613 005, Tamil Nadu, India \\ ${ }^{2}$ University College of Engineering Dindigul-624 622, Tamil Nadu, India \\ †Corresponding Author Email: kavithha@gmail.com
}

(Received May 15, 2018; accepted July 18, 2018)

\begin{abstract}
Desirable thermal properties of nanofluid is the vital reason for using nanofluid. There is an exemplary development in various applications using nanofluid. Mathematical and experimental models were developed to predict the thermal properties of nanofluids, the models are tiresome and expensive and have discrepancies between them. Soft computing tools are most useful in prediction, classification and clustering the data with good accuracy and with less expensive. In this paper, comparative analysis of Multi Layer Perceptron (MLP) model and Support Vector Regression (SVR) model were done by using various evaluation criterions. The two models developed to predict the thermal conductivity ratio of $\mathrm{CNT} / \mathrm{H}_{2} \mathrm{O}$ and the results were compared. The present modeling has been carried out using MATLAB $2017 \mathrm{~b}$. In both the models, the experimental values and predicted values possess good accordance. Regression coefficient value (R2) for overall data is 0.99 and 0.98 for MLP and SVR models respectively. The Root Mean Square Error (RMSE) value is less in MLP model when compared with SVR model, RMSE values are 0.01578 and 0.01812 respectively. The prediction is best in MLP model but with limited experimental data set, it fails to address over fitting problem, whereas SVR model is ideal with limited data set, it overcomes over fitting problem and possess better generalization than MLP model.
\end{abstract}

Keywords: Nano fluids; Thermal conductivity; Artificial neural network; Multilayer perceptron; Support vector regression.

\section{INTRODUCTION}

Nanofluid has succeeded in various fields. The wide range starts from technical applications like automotive sector, solar thermal applications, geo thermal applications, nuclear applications to bio-medical applications. The effectiveness of nanofluid depends upon various thermo-physical properties such as thermal conductivity, viscosity and heat transfer coefficient. Theoretical models and experimental models were developed to predict thermal conductivity of nanofluid. A prediction by experimental model is expensive and also correlations between theoretical and experimental models are little. The difficulties exist between these models were addressed by using soft computing tools. Soft computing tools are useful in prediction, classification and clustering of data from large databases using machine learning algorithms. Machine learning algorithms types are supervised learning, unsupervised learning, semi supervised learning, reinforcement learning, transduction and learning to learn. The utility of algorithm is to discover the accurate pattern reside in the large data set.
Artificial neural network is a parallel processing network, it organize the non linear relationship between response and explanatory variables. Multilayer Perceptron is a feed forward artificial neural network, which consist of three layers namely input layer, hidden layer and output layer. MLP neural network use back propagation methodology to change the weight in proportion to the error between the desired output and predicted output. Support vector algorithm is a non-linear generalization algorithm based on statistical learning theory, familiarly called as VC theory. In SVR model, the set of training data includes independent variables and observed dependent variables. The goal of SVR model is to find the predict function from the observed function with a minimal error values and to exhibit excellent generalization. Dataset comprise of 132 samples of $\mathrm{CNT} / \mathrm{H}_{2} \mathrm{O}$ nanofluid are used in this paper to predict the thermal conductivity ratio of the nanofluid. The purpose of this research paper is to do comparative analysis between MLP model and SVR model based on various evaluation criterions. 


\section{ABOUT NANOFLUIDS}

The abundant growth of nanofluids is due to its thermal -physical properties. The thermal conductivity depends on various factors like particle size, particle shape, base fluid, $\mathrm{pH}$ value, temperature, particle volume concentration, particle materials, and thermal conductivity of base materials by Tajik (2013). Temperature, volume fraction and nanoparticle size are the most important properties used to enhance the thermal conductivity of nanofluids Manna (2012), Selvam (2016), Murshed (2008) and Mohammed Reza (2015). Increase in particle volume fraction in MWCNT/ $\mathrm{H}_{2} \mathrm{O}$ sequentially increase heat transfer rate and increases pressure drop stated by Sulochana (2016), similarly increasing in the solid volume fraction increases the performance of heat transfer in the $\mathrm{Al}_{2} \mathrm{O}_{3}$ nanofluid stated by Uddin (2017).

$\mathrm{Al}_{2} \mathrm{O}_{3} / \mathrm{H}_{2} \mathrm{O}$ nanofluid was used and experimented in shell and coiled tube heat exchangers and revealed that heat transfer rate is high in nanofluids when compared with conventional heat transfer fluids. Beck (2010) through experiment concluded that increase in temperature, increases the thermal conductivity of nanofluids. Thermal conductivity of nanofluid increases with size of nanoparticle stated by Naramgari (2016). The researcher examined MWCNT/ $\mathrm{H}_{2} \mathrm{O}$ nanofluid through experiment and stated that, brake thermal efficiency increases significantly in nanofluid than in water when it is used as a coolant in a four stroke diesel engine by Shakeri (2016). The stability of heat transfer of MWCNT with seven different base fluids were studied by Salehi (2011) and stated that MWCNT with grown oil possess good stability.

Spherical shaped nano particles has better thermal enhancement in of $\mathrm{CuO} / \mathrm{H}_{2} \mathrm{O}$ and $\mathrm{Al}_{2} \mathrm{O}_{3} / \mathrm{H}_{2} \mathrm{O}$ nanofluids than cylindrical shaped nano particles stated by Vaferi (2014). The heat transfer rate of $\mathrm{CuO} / \mathrm{H}_{2} \mathrm{O}$ and $\mathrm{Al}_{2} \mathrm{O}_{3} / \mathrm{H}_{2} \mathrm{O}$ nanofluids increase with small sized nano-particles revealed by Ariama (2015) and Masound (2017). The effectiveness of the binary nanofluid is higher than that with the base fluid by Ali (2013). The mass transfer rate of the dusty nanofluid increases with chemical reaction parameter and by fluid particle interaction parameter experimented and stated by Akbar (2014). Venkatesh et al. (2016) studied the flow of heat and fluid in solar air heater using Computational Fluid Dynamics (CFD). The predicted temperature results were observed within a deviation of $\pm 10.64 \%$.

Enrichment in heat transfer rate of nanofluids made to develop various applications start from automotive sectors to cancer therapeutic applications. From the literature review, it is observed that prediction of thermal conductivity of nanofluid is essential to develop more applications using nanofluid in wider range. A very little work has been done on finding the pattern of thermal properties of nanofluids, therefore in this paper, soft computing tools are used to exhibit the pattern of thermal properties of nanofluids and the performance of the developed models were compared.

\section{DESCRIPTION OF MLP MODEL}

Machine learning system is the fundamental to artificial neural networks, fuzzy systems, simulated annealing, rough sets, support vector machines and genetic algorithms to learn and predict the hidden patterns from the data sets. Artificial neural network (ANN) is capable network, to control the nonlinear relationship between independent variables and dependent variables Ibrahim (2018). Multi-layer feed forward layer neural network consist of minimum three layers namely input layer, hidden layer and output layer. The network changes its weight in proportion to the error between the desired output and predicted output by back propagation.

Fig.1. depicts, simple MLP - neural network model, the input layer, which comprise of explanatory variables, Temperature $\left(\mathrm{T}\right.$ in $\left.{ }^{\circ} \mathrm{C}\right)$ and Volume fraction $(\varphi)$, the output layer, consist of response variable, thermal conductivity ratio and hidden layer where the number of neurons and weight between neurons are adjusted to get the desired output.

Thermal efficiency, thermal resistance and thermal conductivity experimental values are compared respectively with MLP predicted values, it was found, both the models possess good agreement between them stated by Vapnik (1999) and Mohammed (2016). Srinivasan (2017) studied and analysed about the missile with grid fins and the effect on flow drag using ANSYS. Godwin (2017) and investigated about the optimum parameters for obtaining the best performance using alternate fuels of IC engines working under the current cooling system using Nanofluids.

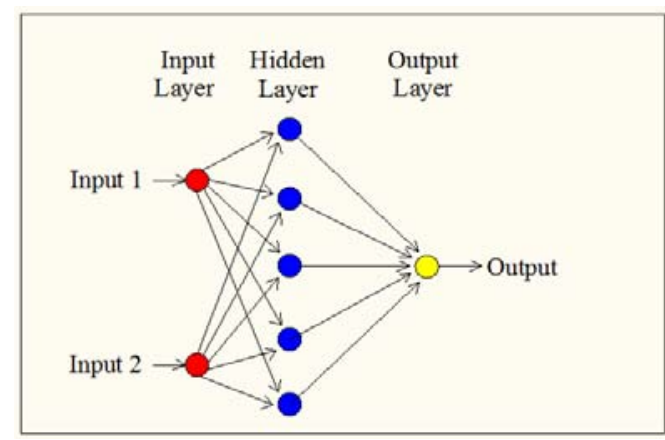

Fig. 1. Representation of simple MLP Model.

\section{DESCRIPTION OF SVR MODEL}

Support vector regression is a computational nonlinear generalization algorithm using statistical learning theory, familiarly called as VC theory. It is stated that, for water distribution system management the SVR methodology performed better in generalization than ANN method. It was revealed that prediction of thermal conductivity of nanofluid by using support vector regression model 
is accurate

In SVR model, the set of training data includes independent variables and observed dependent variables. The objective of SVR model is to map the inputs in a high dimensional feature space using nonlinear transformation mapping function by inner product function and forming linear regression in the high dimensional space.

In fig.2. regression curve with data points fitted by SVR is shown. SVR algorithm finds a function that approximate the observed response value and predicted response value with a precision $s$ value, errors lesser than $\varepsilon$ are insignificant. Let set of data is $\left(\mathrm{x}_{\mathrm{n}}, \mathrm{y}_{\mathrm{n}}\right)$, where $\mathrm{x}_{n}$ is the vector of explanatory variables, $\mathrm{y}_{n}$ is the definite value of response variable, $\mathrm{n}$ takes the value from 1 to $\mathrm{N}$, where $\mathrm{N}$ is the total number of data pairs. The linear model equation can be written as in Eq.(1).

$$
f(x, \omega)=w \cdot x+b
$$

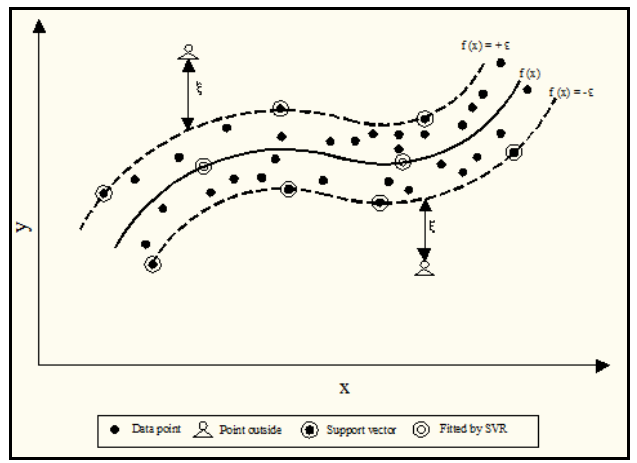

Fig. 2. Optimal hyper plane with slack variables.

Where w, b represents coefficients and constant. The quality of estimation is evaluated by using $\boldsymbol{\varepsilon}$ insensitive loss function, it is written as in Eq.(2).

$L_{\varepsilon}(y, f(x, \omega))=\max (|y-f(x, \omega)|-\varepsilon, 0)$

The minimizing the regularized risk function is given as in Eq.(3).

$\operatorname{Min} . R\left(w, \xi_{i}^{*}\right)=\frac{1}{2}\|w\|^{2}+C \sum_{i=1}^{n}\left(\xi_{i}-\xi_{i}^{*}\right)$

subject to:

$$
\begin{cases}y_{i}-<w, x_{i}>-b & \leq \varepsilon+\xi_{i} \\ <w, x_{i}>+b-y_{i} & \leq \varepsilon+\xi_{i}^{*} \\ \xi_{i}, \xi_{i}^{*} \geq 0 & \end{cases}
$$

$\mathrm{C}$ term is trade off the complexity, it determines the trade off between the model flatness and empirical risk. This optimization problem can be transformed in to dual problem by applying kernel trick for nonlinear case, it is shown in Eq.(4)

$$
\left\langle\phi\left(x_{i}\right), \phi(x)\right\rangle=K\left(x_{i}, x\right)
$$

The solution for optimization problem is given as in
Eq.(5)

$$
f(x)=\sum_{i=1}^{n_{S V}}\left(\alpha_{i}-\alpha_{i}^{*}\right) K\left(x_{i}, x\right)
$$

subject to: $0 \leq \alpha_{i}^{*} \leq C, 0 \leq \alpha_{i} \leq C$

where $n_{\mathrm{SV}}$ is the number of support vectors and $K\left(x_{i}, x\right)$ is a kernel function.

The common kernel functions are Linear, Gaussian kernel otherwise called as Radial Basis Function (RBF) kernel and Polynomial kernel. The three kernel functions are expressed in the Eq. (6), (7), (8) respectively.

Linear Kernel: $K\left(x_{i}, x_{j}\right)=x_{j}^{T} x_{i}$

Radial Basis Kernel:

$$
K\left(x_{i}, x_{j}\right)=\exp \left(-\frac{1}{2 \sigma^{2}}\left\|x_{i}-x_{j}\right\|^{2}\right)
$$

Polynomial Kernel:

$$
K\left(x_{i}, x_{j}\right)=\left(x_{i}^{T} x_{j}+1\right)^{d}
$$

Prediction of thermal conductivity of nanofluid by experiment is expensive. Finding an accurate model with a limited dataset is disadvantageous using MLP model due to its generalization behavior, SVR model possess better generalization than MLP model even with fewer data set. A very few researchers were worked with SVR model to predict the thermal conductivity of nanofluid, hence in this paper, SVR model were deployed to predict the thermal conductivity of CNT/ $\mathrm{H}_{2} \mathrm{O}$ and to exhibit the generalization ability of SVR.

\section{EVALUATION CRITEROIN}

In this paper, a number of evaluation criterions are used to evaluate the MLP model and SVR model. These criteria are applied to measure the accordance between experimental values and predicted values. The criterions are Mean Square Error (MSE), Root Mean Square Error (RMSE), Normalized Mean Square Error (NMSE), Mean Absolute Error (MAE) and Regression coefficient value $\left(\mathrm{R}^{2}\right)$. The mathematical formulation of criterions are shown in Eq. (9),(10),(11),(12) and (13) respectively.

$$
\begin{aligned}
& M S E=\frac{1}{n} \sum_{i=1}^{n}\left(k_{p}-k_{a}\right)^{2} \\
& R M S E=\sqrt{\frac{1}{n} \sum_{i=1}^{n}\left(k_{p}-k_{a}\right)^{2}} \\
& N M S E=\frac{M S E}{\operatorname{var}\left(k_{a}\right)} \\
& M A E=\frac{1}{n} \sum_{i=1}^{n}\left|k_{a}-k_{p}\right| \\
& R^{2}=1-\frac{\sum_{i=1}^{n}\left(k_{a}-k_{p}\right)^{2}}{\sum_{i=1}^{n}\left(k_{a}-\bar{k}_{a}\right)^{2}}
\end{aligned}
$$


Where $\mathrm{k}_{\mathrm{p}}, \mathrm{k}_{\mathrm{a}}$ denotes thermal conductivity ratio of predicted data and experimental data, $\bar{k}_{a}$ is mean value of thermal conductivity of experimental data for ' $n$ ' data values, ' $n$ ' denotes total number of data samples.

\section{Results AND Discussion}

The experimental values used for training the MLP model and SVR model have been taken from the paper [32]. Taken 132 sample data set comprise of temperature, volume fraction and thermal conductivity ratio of $\mathrm{CNT} / \mathrm{H}_{2} \mathrm{O}$ to train the models. Many researchers in the literature review revealed the importance of temperature and volume fraction thermo-physical properties in enhancing the thermal conductivity of nanofluid. In both the models, temperature and volume fraction are taken as input in other words as explanatory variables and thermal conductivity ratio as response variable.

To show the importance of neurons in training the MLP, the network modeled, by varying neurons from five to twenty in hidden layer and Levenberg Marquardt (LM) training algorithm is implemented. The performance of MLP network with a range of neurons in hidden layer is shown in Table 1 . The range between 10 and 15 neurons are having less mean square error value when compared with 5 and 20 neurons. Overall regression coefficient (R2) is higher in 15 and 10 hidden neurons with 0.99216 and 0.99043 values respectively it represented in Fig. 3 and in Fig. 4

Multilayer Perceptron (MLP) model predicts the thermal conductivity ratio accurately and it has good agreement with experimental values, it is represented in Fig. 5 .

SVR model the set of training data includes independent variables and observed dependent variables. The objective of SVR model is to map the inputs in a high dimensional feature space using nonlinear transformation mapping function by inner product function and forming linear regression in the high dimensional feature space. SVR model with different kernel function namely linear kernel, Gaussian or RBF kernel and polynomial kernel were used to train the model without any test cross validation. The results obtained are shown in Table 2.

SVR model with cross validation is introduced to protect the data against over fitting problem. The cross validation method, partition the data set in to number of folds and provide the estimated accuracy in each piece of data set fold. The linear kernel, Gaussian kernel with various kernel scales of 0.35 , 1.4 and 5.7 are used along with polynomial order 2 and 3, their results were shown in Table. 3.

Among three different kernel functions, polynomial kernel with third order function performs better by having lesser root mean square value (RMSE) compared with other kernel functions. In Table 4, experimental values are compared with the predicted values by three kernel functions and it is revealed that the values predicted by polynomial kernel where very close to the experiment values.

Fig.6, Fig.7 and Fig.8 depicts the good agreement between the experimental values and predicted values by linear kernel function, Gaussian kernel function and polynomial kernel function and it is clearly shown that prediction by polynomial function is more accurate.

Table 1 MLP model with range of hidden neurons

\begin{tabular}{|c|c|c|c|c|c|}
\hline $\begin{array}{c}\text { No.of } \\
\text { Hidden } \\
\text { Neurons }\end{array}$ & MSE & RMSE & NMSE & MAE & $R^{2}$ \\
\hline 5 & 0.000349 & 0.018682 & 0.000286 & 0.014402 & 0.99031 \\
\hline 10 & 0.000273 & 0.0165223 & 0.000224 & 0.014739 & 0.99043 \\
\hline 15 & 0.000249 & 0.01578 & 0.000204 & 0.015635 & 0.99216 \\
\hline 20 & 0.000403 & 0.020075 & 0.00033 & 0.01324 & 0.98854 \\
\hline
\end{tabular}

Overall R $=0.99216$

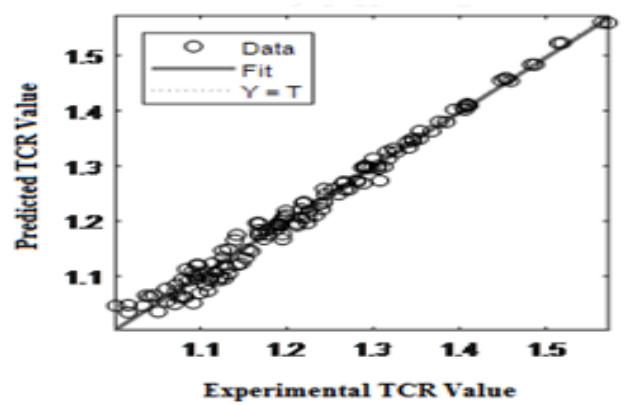

Fig. 3. Overall Regression values for 15 Hidden neurons

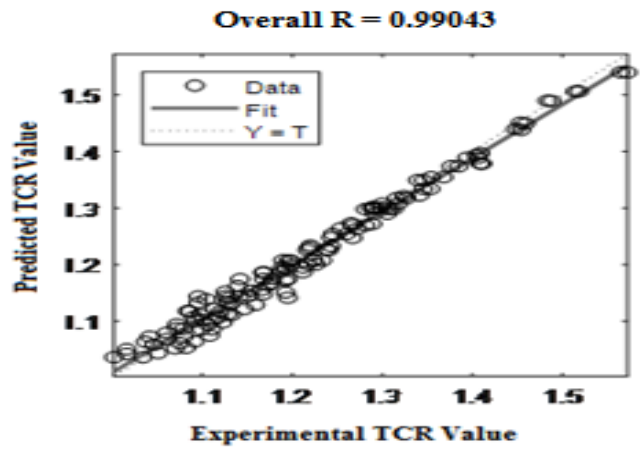

Fig. 4. Overall Regression values for 10 Hidden neurons 
R. Kavitha and P. C. Mukesh Kumar/JAFM, Vol. 11, Special Issue, pp. 7-14, 2018.

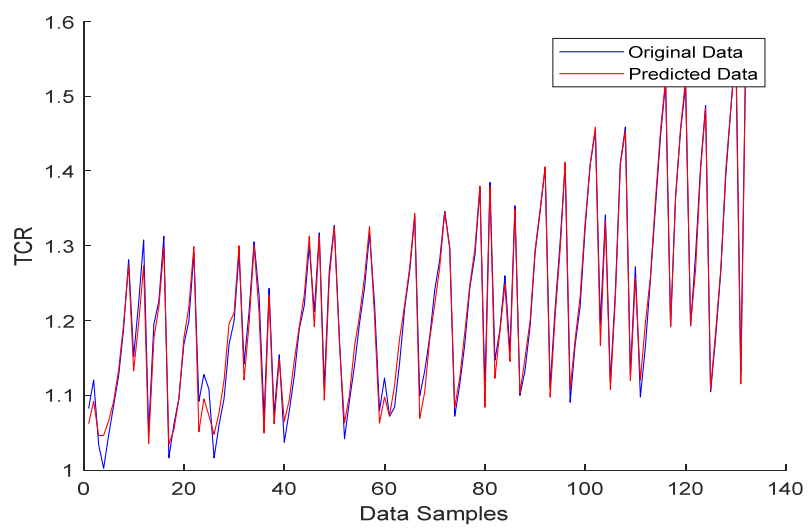

Fig. 5. Experimental data and predicted data using MLP

Table 2 SVR-Model with different kernel function

\begin{tabular}{|c|c|c|c|c|c|}
\hline SVRMODEL & MSE & RMSE & NMSE & MAE & $\mathrm{R}^{2}$ \\
\hline Linear & 0.001954 & 0.044202 & 0.001602 & 0.035374 & 0.88 \\
\hline Gaussian & 0.000345 & 0.018573 & 0.000283 & 0.015069 & 0.98 \\
\hline Polynomial (3rd Order) & 0.000282 & 0.01679 & 0.000231 & 0.013765 & 0.98 \\
\hline
\end{tabular}

Table 3 SVR with Cross - Validation of 5 fold

\begin{tabular}{|c|c|c|c|c|c|c|}
\hline SVR.MODEL & MSE & RMSE & MAE & $\mathrm{R}^{2}$ & $\begin{array}{c}\text { Speed } \\
(\mathrm{Obs} / \mathrm{Sec})\end{array}$ & $\begin{array}{c}\text { Training Time } \\
(\mathrm{Sec})\end{array}$ \\
\hline Liner & 0.0021327 & 0.046181 & 0.037412 & 0.87 & 1300 & 5.5743 \\
\hline $\begin{array}{c}\text { Gaussian } \\
\text { KS-0.35 }\end{array}$ & 0.0010513 & 0.032425 & 0.025289 & 0.94 & 8200 & 0.90408 \\
\hline $\begin{array}{c}\text { Gaussian } \\
\text { KS-1.4 }\end{array}$ & 0.00053523 & 0.023135 & 0.018257 & 0.97 & 7300 & 0.9355 \\
\hline $\begin{array}{c}\text { Gaussian } \\
\text { KS-5.7 }\end{array}$ & 0.0018449 & 0.042952 & 0.034625 & 0.89 & 5700 & 0.95332 \\
\hline $\begin{array}{c}\text { Polynomial } \\
\text { (2nd Order) }\end{array}$ & 0.0009608 & 0.030997 & 0.024989 & 0.94 & 10,000 & 6.7719 \\
\hline $\begin{array}{c}\text { Polynomial } \\
\text { (3rd Order) }\end{array}$ & 0.0003286 & 0.018126 & 0.014791 & 0.98 & 7600 & 1.6904 \\
\hline
\end{tabular}

Table 4 SVR Model Cross validation with 5 fold

\begin{tabular}{|c|c|c|c|}
\hline \multirow{2}{*}{ Experimental Value } & \multicolumn{3}{|c|}{ Table 4 SVR Model Cross validation with 5 fold } \\
\cline { 2 - 4 } & $\begin{array}{c}\text { Linear } \\
\text { Kernel }\end{array}$ & $\begin{array}{c}\text { Gaussian } \\
\text { Kernel }\end{array}$ & $\begin{array}{c}\text { Polynomial } \\
\text { Kernel }\end{array}$ \\
\hline 1.2238 & 1.1948 & 1.2058 & 1.2022 \\
\hline 1.0703 & 1.0639 & 1.0608 & 1.0476 \\
\hline 1.1735 & 1.1390 & 1.1481 & 1.1565 \\
\hline 1.0932 & 1.1131 & 1.1007 & 1.0969 \\
\hline 1.4534 & 1.4423 & 1.4711 & 1.4590 \\
\hline 1.4480 & 1.3735 & 1.4509 & 1.4432 \\
\hline
\end{tabular}




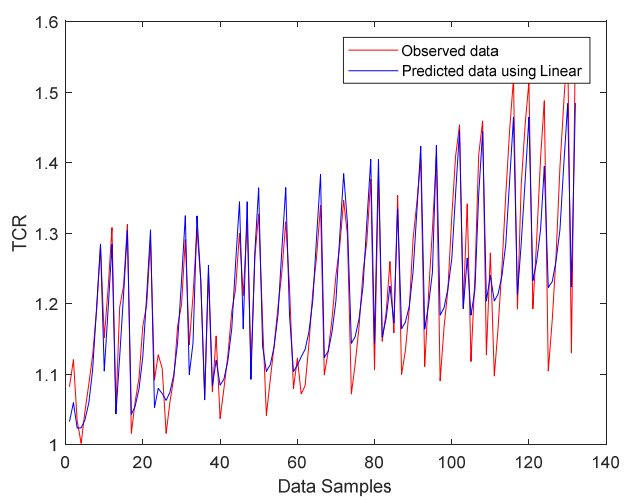

Fig. 6. Prediction using SVR-Linear Kernel Model

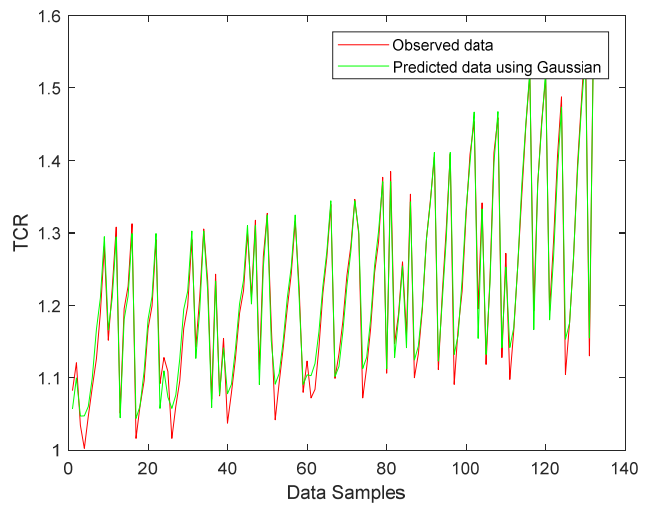

Fig. 7. Prediction using SVR-Gaussian Kernel Model

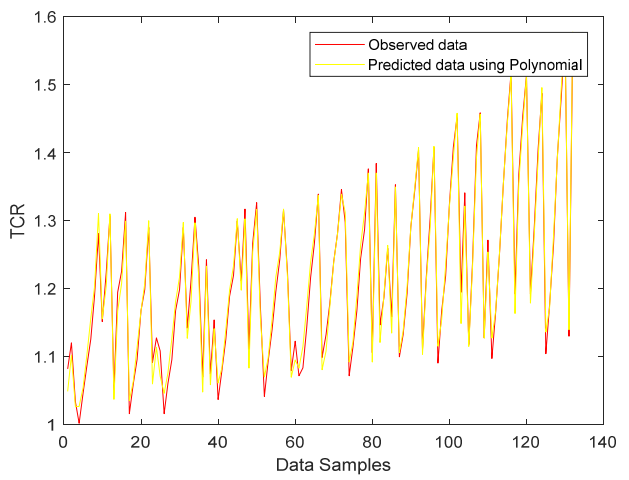

Fig. 8. Prediction using SVR-Polynomial Kerne Model
In Table 5, the MLP model and SVR model performance were compared. MLP with 15 neurons has been chosen as its performance is better than 5, 10, 20 neurons in the hidden layer. SVR model trained using polynomial kernel function, without cross validation and including cross validation were taken and compared the performance. Among all the developed models the least RMSE value is achieved by MLP model but, for generalization purpose and to overcome over fitting problem with limited data set of nanofluids, SVR model is preferred due its generalization ability even with fewer data sets.

\section{Conclusion}

In this research paper, comparative analysis of Multi Layer Perceptron (MLP) model and Support Vector Regression (SVR) model were done by using various evaluation criterions. The two models developed to predict the thermal conductivity ratio of $\mathrm{CNT} / \mathrm{H}_{2} \mathrm{O}$ and the results were compared. Both the models possessed a good accordance between experimental values and predicted values. Mean Square Error (MSE), Root Mean Square Error (RMSE), and Regression coefficient value $\left(\mathrm{R}^{2}\right)$ are considered as main evaluation criterions. Regression coefficient value from the best MLP model and SVR model are obtained, the values are 0.99 and 0.98 respectively, the coefficient value is closer to 1 , hence it could be implied that the predicted results are reliable and accurate. The Root Mean Square Error (RMSE) value is lesser in MLP model when compared with SVR model, the values are 0.01578 and 0.01812 respectively. To predict the model, we required large data set, in the case of prediction of thermal conductivity, we required more experimental values that is too expensive. With limited dataset, MLP model suffers in over fitting problem and in generalization to conquer this problem SVR model is preferred to predict the thermal conductivity of nanofluid and to exhibit excellent generalization behavior with fewer data.

Table 5 Comparison between developed models

\begin{tabular}{|c|c|c|c|c|}
\hline Developed Model & MSE & RMSE & MAE & $\mathrm{R}^{2}$ \\
\hline MLP-15 Neurons & 0.000249 & 0.01578 & 0.015635 & 0.99216 \\
\hline $\begin{array}{c}\text { SVR Polynomial } \\
\text { Kernel Without Cross } \\
\text { Validation }\end{array}$ & 0.000282 & 0.01679 & 0.013765 & 0.98 \\
\hline $\begin{array}{c}\text { SVR Polynomial } \\
\text { Kernel With Cross } \\
\text { Validation }\end{array}$ & 0.0003286 & 0.018126 & 0.014791 & 0.98 \\
\hline
\end{tabular}




\section{REFERENCES}

Alade, I. O., T. A. Oyehan, I. K. Popoola, Sunday O. Olatunji, O. Olatunji and B. Aliyu (2018) Modeling thermal conductivity enhancement of metal and metallic oxide nanofluids using support vector regression, Advanced Powder Technology 29 (1) 157-167.

Ariana, M. A., B. Vaferi. and G. Karimi. (2015) Prediction of Thermal conductivity of alumina water-based Nanofluids by Artificial Neural Networks, Powder Technology, 278 1-10.

Armou, S., R. Mir, Y. El Hammami, K. Zine-Dine and M. El Hattab (2017) Heat and Mass Transfer Enhancement in Absorption of Vapor in Laminar Liquid Film by Adding NanoParticles, Journal of Applied Fluid Mechanics 10 (6), 1711-1720.

Battira, M., R. Rachid Bessaïh, Radial and Axial (2016)Magnetic Fields Effects on Natural Convection in a Nanofluid-filled Vertical Cylinder, Journal of Applied Fluid Mechanics 9 (1), 407-418.

Beck, M., Y. Yuan, P. Warrier and A. Teja (2010) The effect of particle size on the thermal conductivity of alumina nanofluids in water,ethylene glycol and ethyleneglycol with water mixtures, Journal of Nanoparticles Research 12, 1469-1477.

Godwin Antony, A., S. Dinesh, K. Rajaguru and V. Vijayan (2017) Analysis and Optimization of Performance Parameters in Computerized I.C. Engine Using Diesel Blended with Linseed Oil and Leishmaan's Solution, Mechanics and Mechanical Engineering 21(2), 193-205.

Hemmat Esfe, M., K. Motahari, E. Sanatizadeh, M. Afrand, H. Rostamian and M. R. Hassani Ahangar, (2016) Estimation of thermal conductivity of CNT-water in low temperature by artificial neural network and correlation", International Communications in Heat and Mass Transfer 76, 376-381.

Hojjat, M., S. Gh. Etemad. R. Bagheri, and J. Thibault (2011) Thermal Conductivity of nonNewtonian nanofluids: Experimental data and modeling using neural network, International Journal of Heat and Mass Transfer 54, 10171023.

Jamal, T., Abadi. M. and Zam Zamian A. H, (2013) Optimization of Thermal Conductivity of A12O3 Nanofluid by Using ANN and GRG Methods, International Journal of Nanoscience and Nanotechnology 9 (4), 177-184.

Khajeh, K., L. Jahanshaloo, S. Ebrahimi and H. Aminfar (2018) Mixed Convection Heat Transfer of A12O3 Nanofluid on the Elliptical Shapes: Numerical Study of Irreversibility, Journal of Applied Fluid Mechanics 11 (1), 177-189.

Lakshmanan, P., P. Kaliyappan, M. Ranjithkumar,
K. Aravinth, D. Vakkachan, C. Moorthy and S. Kumar, (2017) An Experimental Investigation to Study the Performance and Emission Characteristics of Chicken Fat Oil Fuelled DI Diesel Engine, Journal of Applied Fluid Mechanics10 (Special Issue), 85-91

Liu, M. S., M. C. C. Lin, C. Y. Tsai and C. C. Wang (2006) Enhancement of thermal conductivity with $\mathrm{Cu}$ for nanofluids using chemical reduction method, International Journal of Heat Mass Transfer 49 (17-18), 3028-3033.

Manna, O., S. K. Singh and G. Paul, (2012) Enhanced thermal conductivity of Nano SiC dispersed water based Nano fluid, Bulletin of Material sciences 35 (5), 707-712.

Murshed, S., M. S. Leong, K. C. Cand Yang, (2008) Thermophysical and Electro kinetic Properties of Nano fluids-A Critical Review, Applied Thermal Engineering 28 (17-18), 2109-2125.

Noghrehabadi, A. and R. Pourrajab (2016) Experimental investigation of forced convective heat transfer enhancement of $\gamma-\mathrm{Al} 2 \mathrm{O} 3 /$ water nanofluid in a tube, Journal of Mechanical Science and Technology 30 (2), 943-952.

Sadollah, A. , A. Ghadimi, I. H. Metselaar and A. Bahreininejad (2013) Prediction and optimization of stability parameters for titanium dioxide nanofluid using response surface methodology and artificial neural networks, Science and Engineering Composite Materials 20 (4) $1-12$.

Salehi. H., S. Zeinali Heris, M. Koolivand Salooki, S. H. Noei (2011) Designing a Neural Network for Closed Thermosyphon with Nanofluid using a Genetic Algorithm, Brazilian Journal of Chemical Engineering 28 (1), 157-168.

Salimpour, M. R. and A. Dehshiri-Parizi, (2015) Convective heat transfer of nanofluid flow through conduits with different cross-sectional shapes, Journal of Mechanical Science and Technology 29 (2), 707-713.

Selvam, C., D. Mohan Lal and H. Sivasankarn, (2016) Thermophysical properties of ethylene glycol-water mixture containing silver nanoparticle, Journal of Mechanical Science and Technology 30 (3), 1271-1279.

Shakeri, A., Ghassemi, M., Hassani, A. Hajian (2016) Investigation of material removal rate an surface roughness in wire electrical discharge machining process for cementation alloy steel using artificial neural network, International Journal of Advanced Manufacturing Technology82 (1-4), 549 - 557.

Shirzad, A., M. Tabesh and R. Farmani (2014) A comparison between performance of support vector regression and artificial neural network in prediction of pipe burst rate in water distribution networks, Journal of Civil Engineers18 (4) 941-948.

Srinivasan, R., V. Vijayan and K. Sridhar (2017) 
R. Kavitha and P. C. Mukesh Kumar/JAFM, Vol. 11, Special Issue, pp. 7-14, 2018.

Computational Fluid Dynamic Analysis of Missile with Grid Fins Journal of Applied Fluid Mechanics 10 (Special Issue), 33-39.

Sulochana, C., N. Sandeep (2016) Flow and Heat Transfer Behavior of MHD Dusty Nanofluid past a Porous Stretching/Shrinking Cylinder at Different Temperatures, Journal of Applied Fluid Mechanics 9 (2), 543-553.

Uddin, Z., R. Asthana, M. K. Awasthi and S. Gupta (2017) Steady MHD Flow of NanoFluids over a Rotating Porous Disk in the Presence of Heat Generation/Absorption: a Numerical Study using PSO, Journal of Applied Fluid Mechanics 10 (3), 871-879.

Vafaei, M., M. Afrand, N. Sina, R. Kalbasi, F. Sourani, H. Teimouri, (2017) Evaluation of thermal conductivity of MgO-MWCNTs/EG hybrid nanofluids based on experimental data by selecting optimal artificial neural networks, Physica E, 85 90-96.

Vaferi, B., F. Samimi, E. Pakgohar and D. Mowla, (2014) Artifical neural network approach for prediction of thermal behaviour of nanofluids flowing through circular tubes, Powder Technology, 267 1-10.

Vapnik, V. (1999) The nature of statistical learning theory, $2^{\text {nd }}$ edition Springer.

Venkatesh, R. and V. Vijayan (2016) Performance Evaluation of Multipurpose Solar Heating System, Mechanics and Mechanical Engineering 20(4), 359-370. 